\title{
Transfer securing of vocational education
}

\author{
Kaethe Schneider ${ }^{1,2}$ \\ ${ }^{1}$ Institute for Education and Culture, Jena, Germany \\ ${ }^{2}$ University of Jena, Jena, Germany
}

\section{Email address:}

k.schneider@uni-jena.de

\section{To cite this article:}

Kaethe Schneider. Transfer Securing of Vocational Education. Science Journal of Education. Vol. 1, No. 3, 2013, pp. 36-42. doi: $10.11648 /$ j.sjedu.20130103.12

\begin{abstract}
The objective of the study is to examine the extent to which shortcomings can be identified in the sub-process chain for securing and evaluating the learning transfer in vocational education at companies in the Federal Republic of Germany. It is argued that there are shortcomings in securing the learning transfer in vocational education at companies in Germany. All the companies in the DAX30, MDAX, SDAX and TecDAX and/or the 500 family-owned companies with the highest sales revenue and at least 1,000 employees were surveyed in this investigation. The sampling consisted of 632 of 660 companies in total. 107 companies took part in the online survey. The findings in the study show that only one quarter of the examined companies in Germany secured and evaluated the entire process chain for the learning transfer. The methods applied for securing the learning transfer at companies exhibit conceptual shortcomings in the securing of the learning transfer. A model for securing the learning transfer has been developed and recommended for specific transfer securing of vocational education for German companies.
\end{abstract}

Keywords: Learning Transfer, Vocational Education, Learning Transfer Securing

\section{Introduction}

Sustainable vocational education at companies makes a contribution to corporate success and to the ongoing development of employees in a company. Vocational education in companies should enable the application of learned material in a functional field.

Measures for securing the learning transfer in professional vocational education are regarded as ineffective: 90 percent of the material learned in vocational education measures has no impact on the employees' responsibilities at the workplace [7]. The low effectiveness of vocational education at companies has also been found in Anglo-Saxon studies [14]. Reference [19] assumes that 60 to 90 percent of the learned material is not applied on the job.

The term learning transfer describes the application of learned material to a new situation [12]. "Vocational education is understood to include vocational education measures that consist of pre-planned organized learning and are fully or partially financed by companies for their employees" [25].

The programs for securing the learning transfer are not efficient if companies invested $€ 28.6$ billion in vocational education in 2010 [22] and if it is assumed that only 10 percent of the learned material is used in the work context. $€ 8.7$ billion of the investments are attributed to direct vocational education costs, $€ 17.3$ billion to indirect vocational education expenses and $€ 2.7$ billion to the costs for the vocational education personnel [22]. Roughly $€ 25$ billion, which is invested in vocational education at companies in Germany, would not be productive as a result.

It is argued that there are shortcomings in securing the learning transfer in vocational education at companies in Germany. The objective of this study is to investigate what are the shortcomings in the sub-processes chain for securing learning transfer in companies and what theoretical model could be designed to improve the processes chain for German companies.

There are only a few studies in which the actual situation regarding measures for securing the learning transfer at companies were available which made it difficult for a critical literature review process. The European Continuing Vocational Training Surveys, which are also regularly conducted in all the member states of the European Union, include roughly 10,000 companies in Germany with 10 or more employees from almost every industry in their examinations. In these surveys, a question about quality assurance is asked: 46.9 percent of the surveyed companies in Germany measure the satisfaction of the participants in a questionnaire, 59.1 percent evaluate the behavior or the performance of the participants with regard to vocational education objectives and 43.9 percent evaluate or measure the impact of the vocational education on the performance 
of certain departments or the entire company [25]. The evaluation and measurement of the impact varies in relation to the employment size classes: The larger the company, the smaller the percentage of companies that evaluate or measure the impact [25].

According to a study conducted and reported by the American Society for Training and Development,revealed that only twenty one $(21 \%)$ percent of organizations in the United States of America (USA) were interested in an evaluation of the learning transfer [1].

Studies in which the methods for evaluating the learning transfer in companies are surveyed include questions referring to the different forms of evaluation for ensuring quality. Reference [3], in cooperation with Chalgaf, examined the measures for the evaluation of training effectiveness in Tunisian companies. On the basis of these results, the achievement of the learning objectives and learning satisfaction are evaluated directly after the training.

Reference [8] documents the relatively low importance attached to securing the learning transfer in German companies as compared to quality assurance. Only 10.1 percent of 410 German vocational education companies implemented measures for securing the learning transfer in 2007.

The quality of the measures for securing the learning transfer was examined by references $[2,15,16]$ indirectly with the aid of the model reference [2] developed for judging the beneficial and inhibiting factors for the learning transfer. Since the quality of the measures is determined by a subjective assessment, this may be an inexpensive and efficient method, but it does not reflect an objective point of view with regard to quality assurance.

The understanding of the quality of measures for securing the learning transfer is limited, since the criteria for the evaluation of the measures are insufficiently operationalized. There are only a few studies in which measures for securing the learning transfer were empirically tested as specific learning objectives for sustainable promotion [6]. The first methods are available for evaluations related to the measures for the promotion of complex cognitive goals [21]. On the other hand references [23] argue that securing learning by transferring knowledge was evaluated primarily for measures in acquiring simple skills of workers in companies.

The understanding of learning transfers also differsto the content and the context[18]. Regarding the content the form of the transferred knowledge has to be analyzed. The differences relating to the context refer to the extent of the novelty of a situation.

The process chain for securing the learning transfer is analyzed here at companies in the Federal Republic of Germany and a model for securing the learning transfer in vocational education at companies is outlined.

\section{Methodology}

In the present study, a process model is used to represent the measures for securing the learning transfer. A spiral model for securing the learning transferis developed.
Thismodel is a synthesis of the model for explaining intentional stable behavior [24] and the refusal to change is addressed in relation to the model according to reference [9].

I employ this paradigm for the study because learning transfer results in intentional stable changes and needs to overcome the barriers for changes of the self.

The process chain includes the instruments before, during and after the vocational education measure [4, 5]. The core processes in securing the learning transfer reflect and are designed for planning, implementation and evaluation [26]. Reference [4] recommended practical training transfer strategies for the sub-processes, but not on the basis of a theoretical concept [10].

\subsection{Study Objective}

The lack of a theoretical phase model for securing the learning transfer means that the procedure in the examination is inductive. The objective is to examine the extent to which shortcomings can be identified in the sub-processes for securing and evaluating the learning transfer in vocational education at companies. It is argued that there are shortcomings in securing the learning transfer in vocational education at companies in Germany.

To address this question, I examine the process chain for securing the learning transfer in German companies in order to discover shortcomings and outline a theoretical model that can be used to improve the process chain.

\subsection{Sample and Procedure}

In 2001, this study conducted an investigation on all the companies in the DAX30, MDAX, SDAX and TecDAX and/or the 500 family-owned businesses with the highest sales revenue and at least 1,000 employees were surveyed [20]. The sampling consisted of 632 of 660 companies in total. Twenty eight $(n=28)$ companies were not included in this sample, since the companies refused to participate in the survey after being informed about it by phone or bankruptcy proceedings had been introduced[20]. One hundred and seven $(n=107)$ companies were sampled in the online survey. The response rate was $16.9 \%[20]$.

\subsection{Study Instrument}

The instrument used for collecting the information consisted of a written online survey that contains open-ended and multiple choice questions[20]. The multiple choice questions were answered by using a seven point Likert scale. The project was introduced in a phone call and the questionnaire was sent by email to the line managers in the personnel departments[20]

The object of examination relates to measures for securing the learning transfer at companies in Germany. Whether the companies have methods for securing the learning transfer before, during and after the measure was inquired about in light of the underlying process model. In the case of an affirmative answer, the methods were entered in open-ended questions [20].

The evaluation of the transfer process was broken down in terms of processes according the extent to which the 
target competency is specified before the vocational education measure, the actual competency before and after the vocational education is measured, and the learning transfer performance after the vocational education[20]. In the case of an affirmative answer to one or more of these sub-processes, the applied methods were reported in open-endedquestions[20].

\subsection{Data Analysis}

In order to examine the types of process chains in securing the learning transfer at German companies, the unsorted quantity of objects is divided into groups of similar objects, in this case similar process chains. To identify the groups of companies that are very similar within the groups on the basis of their answers, i.e. companies that select the same category of answers especially frequently, and those that exhibit the greatest differences between the groups, a two-step cluster analysis was conducted. All the items for securing and evaluating the learning transfer were selected as variables in the analysis of the clustering of cases. In the present case they are companies. As compared to the hierarchical method, the two-step cluster analysis has the advantage of allowing objects to also belong to another group in the course of the analysis. This results in a better classification than in the hierarchical methods. The two-step cluster analysis is also more resistant to errors in the assumptions for normal distribution or multinomial distribution [13].

In the first step according to this method, all the objects are clustered in a crude and simplified way. In the second step with a hierarchical cluster analysis, the objects are combined into clusters to be defined more precisely.

\section{Results}

Five clusters were created from the calculation (see Table $1)$.

Table 1.Summary ofclusters

\begin{tabular}{ll}
\hline Algorithm & Two-step \\
\hline Input & 7 \\
Cluster & 5 \\
\hline
\end{tabular}

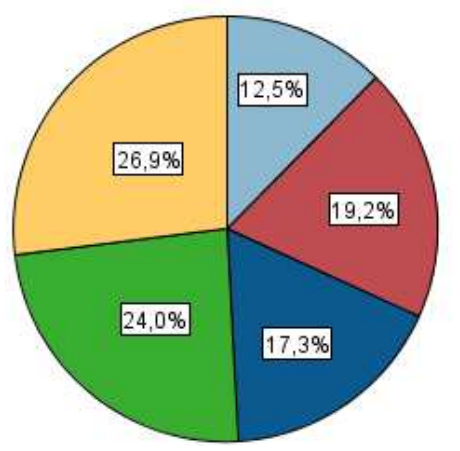

Figure 1. Cluster sizes

The ratio of the largest cluster to the smallest is 2.15 (see Fig. 1).
The answer categories are as follows:

v5 Methods for securing transfer before a vocational education measure

v6 Methods for securing transfer during a vocational education measure

v7 Methods for securing transfer after a vocational education measure

v8 Setting the target competency before the beginning of a vocational education measure

v9 Measuring the actual competency before the beginning of a vocational education measure

v10 Measuring the actual competency after a vocational education measure

v11 Evaluating the learning transfer performance after a vocational education measure

The created clusters are characterized by the following sub-processes (see Fig. 1):

The largest cluster, cluster 5, (28 companies $=26.9 \%)$ shows the companies that use instruments for securing and evaluation across all items. The companies in this cluster usually selected the category "yes" with regard to the application of instruments. The second-largest cluster, cluster 4, $(25$ companies $=24 \%)$ represents the companies that primarily use instruments for securing the learning transfer, but which - across all items - do not use any instruments for evaluating the learning transfer; solely the target competency is determined by just under half of the companies in this cluster. The third-largest cluster, cluster 2 , (20 companies $=19.2 \%$ ) is the cluster where the companies usually do not evaluate or secure the learning transfer across all items. The companies in this cluster select the category "no" for all items with the exception of securing the learning transfer after a measure $(35 \%$ of the companies in this cluster). The second-smallest cluster, cluster 3, (19 companies $=17.3 \%$ ) contains the companies that select the category "yes" for all items in the process chain after vocational education. The smallest cluster, cluster 1, (13 companies $=12.5 \%$ ) is characterized by the fact that in particular the target competency at the beginning of vocational education is specified and the actual competency after vocational education is measured.

According to the selected classification, five characteristic clusters are formed and show different degrees of professionalism:

- $\quad$ Professional cluster (approx. 27 percent): Securing and evaluation of the learning transfer (cluster 5)

- $\quad$ Semi-professional cluster (approx. 24 percent): Securing of the learning transfer (cluster 4)

- Non-professional cluster (approx. 19 percent): Neither securing nor evaluation of the learning transfer (cluster 2)

- $\quad$ Partially professional cluster (17 percent): Securing and evaluation of the transfer after the vocational education measure (cluster 3 )

- Partially professional cluster (13 percent): Determining the target competency in the measurement of competency according to the vocational education measure (cluster 1)

With regard to securing the learning transfer, it is evident that three clusters, which consisted of approximately 50 
(50\%) percent of the responding companies, do not secure the entire chain in the learning transfer process.

The results can be relativized to some extent since the quality of the created clusters is 0.4 and thus in the lower "middle" field (see Fig. 2). 0 would be the same as saying that the individual cases are as far removed from the center of the cluster to which they have been assigned as they are from the center of the other clusters. Accordingly, the classification would not be meaningful. 1 means that the individual cases lie exactly in the center of the cluster to which they have been assigned.

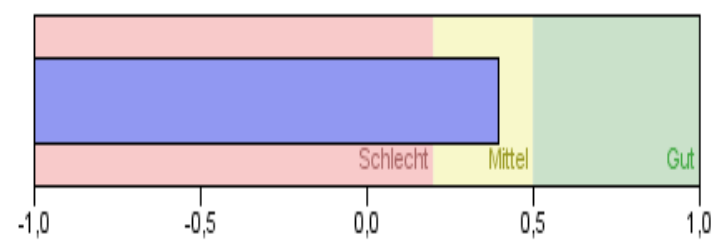

Figure 2. Cluster quality: Silhouette, cohesion and separation values from measurement

The applied measures for securing the learning transfer show shortcomings in the quality of the securing and evaluation of the learning transferquestions [20]:

The questioning of needs and the determination of need in employee meetings $(\mathrm{n}=15)$ were named most frequently as the methods for securing the learning transfer before a measure; the handling of a case $(n=23)$ was reported most often as the method during a measure, and the description of the learned material and its application $(n=18)$ was the most common response after a measure [20]. In the questioning of needs, an employee is asked about his needs; managers and the employee determine the needs in a meeting [20]. In the handling of a case, the practical matters are discussed [20]. The handling of a case in authentic situations is not included in this measure, since it was categorized separately. The measure regarding the description of the learned material and its application reflects the learning results and possibilities for application [20].

Before a measure, the evaluation process is most frequently $(\mathrm{n}=62)$ introduced by setting the target competency that an employee should have [20]. The actual competency is tested by approx. 30\% of the surveyed companies. The most frequent method $(n=19)$ for the evaluation of an employee's learning transfer performance after a vocational education measure was reported to be third-party assessments by managers or other people[20].

\section{Discussion}

It is clear that roughly one quarter of the surveyed companies secures and evaluates the transfer chain with measures, while three quarters of them do not promote the entire learning transfer process with measures. A need for securing the learning transfer in the examined companies in Germany can be affirmed.

The most frequently named measures for securing the learning transfer are not sufficiently characterized by either a concept or the methods for securing the learning transfer.
The questioning of needs is deficient since need as a discrepancy between actual and target competency cannot be recorded validly through oral questioning directly.

As the sole measure, the handling of cases is not sufficient. From constructive didactics, it is known that learning in authentic cases, with regard to the transfer model of situated learning, is conducive to learning transfer and is insufficiently practiced here.

The methods of describing the learned material can also be regarded as insufficient for the transfer requirement, since the learner does not abstract the knowledge - a process that is understood to be required for a transfer according to the transfer model of generalized learning.

The didactic consequences are derived from theory and a model for securing the learning transfer is outlined in the following discussion.

There is no pedagogical model that describes and explains the process for the promotion of the learning transfer. Exploration of the target behavior and the objective-promoting
and objective-impeding actual behavior

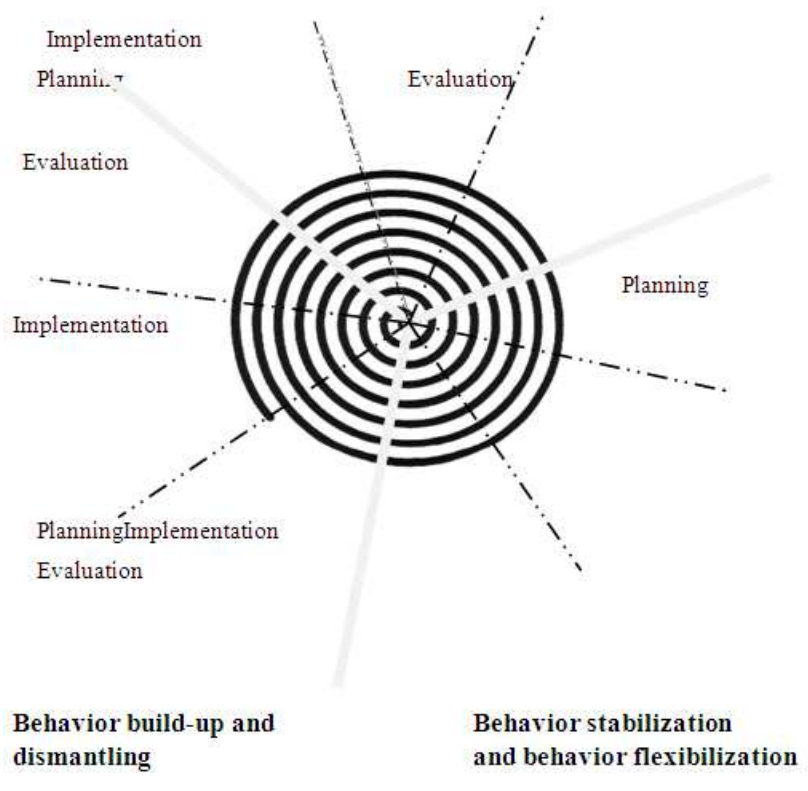

Figure 3. Spiral model for securing the learning transfer (Schneider)

The developed model draft for securing the learning transfer in vocational education at companies represents a spiral model. It understands the transfer process as a repeated process that consists of the sub-processes of planning implementation and evaluation of the methods for securing the learning transfer before, during and after the vocational education measure. The processes of planning, implementation and evaluation repeat themselves cyclically with changing goals and methods until the learning transfer has been completed (see Fig. 3). In a sustainable learning process, intentional stable behavior should be built up and inhibiting behavior modified.

The model represents the processes for building up the desired behavior and for dismantling the objective-impeding behavior. The modification of objective-impeding behavior, which is in competition with the sought learning goals, assumes a central role in securing 
the learning transfer [9].

The intentional stable behavior is described with the aid of the model for explaining intentional stable behavior [24] and the refusal to change is addressed in relation to the model according to reference [9].

Stable intentional behavior results from the interaction of mental and emotional triggers as well as environmental or context-related factors. "The central place of dispositions as the triggers of our intentional and relatively stable behavior is preserved by and represented in the language we use to describe or refer to (1) the relevant behavior, (2) the mental/emotional elements that trigger the behavior, and (3) the underlying conditionals in which the triggers - along with appropriate background conditions - function as antecedent and the behavior functions as consequent" [24].

The refusal to change that is expressed in an objective-impeding behavioral system is explained by reference [9] with the feeling and knowledge system:

The feeling system controls persisting fears. Fears have objectives, hidden competing commitments, as their consequence. The question of why a person stubbornly holds onto a certain way of behavior although this complicates or prevents the achievement of the objective is explained by the hidden competing commitments. Together with the learner, the teacher explores why the person retains the behavior that does not serve the objective. The learner should understand the fears in connection with certain objects, which result from obligations that serve self-confidence, but stand in the way of a change[9].

The knowledge system includes a person's convictions and represents how the world is known and perceived [9]. The reflection on the reasons for the hidden competing commitments allows a person to become aware of their general convictions. The learner explores the principles that form the basis of their obligations. As long as these principles are regarded as true, although they can be true or false, he refuses to change [9]. If the person realizes that there is incoherence between his objectives and personal obligations and major assumptions, he is capable of reflecting on this, questioning it and changing. A change in the major assumptions brings about a reduction in fears on the feeling level [9].

The models show various methods for securing the learning transfer:

Questioning the reasons for broad intentions contributes to the self-determined, sustainable learning "... because descriptions of our desires, beliefs, attitudes, values, and ultimately - our inclinations and dispositions may be all that we have by way of providing a coherent explanation of why we do what we (freely choose to) do" [24]. In dialog, a person discovers which of the emotional and mental factors, in interaction with the context, do not allow the sought behavior to be seen.

"Students can undertake the often complex task of determining the reasons behind a lack of action in a given situation - for example, they may not know how to act, they may not want to act, they may not see the point of acting or realize the opportunity to act, and so on. A common strategy here will be to 'work backward' from behavior ... to uncover the underlying triggers [24]. Together with the adult educator, the learner reflects on the specific situation and the extent to which the lacking ability, motivation, deficient attitude toward the learning objective or the lacking sensitivity to act in the situation are the reason for the failure to behave appropriately. The learning impediments are to be reduced pedagogically and the target behavior is to be built up within the scope of situative learning.

In the case of hidden competing commitments, there is a complexity deficit in the meaning making system [9], which is to be reduced for securing the learning transfer. The complexity of the "meaning making" system can be increased through the experience of productive conflicts so the principles are changed and can be recognized as true or false.

The learning support stretches in spiral form over the entire process of securing the learning transfer before, during and after a measure, and includes the core functions of planning, implementation and evaluation for each sub-process.

Before the measure:

In the analysis of needs, the target and actual behavior is determined before the measure: The actual behavior in specific situations is reflected on with the learner in regard to the abilities, motivation, sensitivity or attitudes as the reasons for shortcomings with respect to the target behavior, and the objective-hindering behavior is explored. The target behavior is specified from the perspective of the learner and organization.

During the measure:

The questioning of principles, the dismantling of learning constraints and the building-up of behavior form the core of the vocational education measure. The teaching perspectives vary during execution and include: the transmission perspective, developmental perspective, apprenticeship perspective, nurturing perspective and social reform perspective, which represent different learning perspectives $[17,23]$.

After the measure:

After the actual measure, the behavior is to be stabilized in the application field and to be made flexible through controlled application in various situations. The process for securing the learning transfer is again taken up, after first passing through the spiral, but from an advanced perspective by exploring new actual-target discrepancies in the activities for the implementation of the acquired behavior.

This model for illustrating the learning transfer in the application field makes it clear that it is insufficient to ensure the securing of the learning transfer only through the acquisition of knowledge.

The personal barriers in learning can be combined with barriers to change in the organization if these questions are reflected on in teams [9].

Such an approach offers a theoretical basis for achieving a deeper understanding of the process for securing the learning transfer in order to promote it effectively.

\section{Conclusions}

It has been argued that there are shortcomings in securing the learning transfer in vocational education at 
companies in Germany. The objective of this study was to investigate what are the shortcomings in the sub-processes chain for securing learning transfer in companies and what theoretical model could be designed to improve the processes chain for German companies.

The results showed that only a minority of the surveyed companiesstates that they secure and evaluate the transfer chain. With regard to the measures of securing the learning transfer it is evident that there are shortcomings as well: The frequently named measures for securing the learning transfer are not sufficiently characterized by a concept or a model.

Based on these deficits a model is developed: It is a spiral model and includes instruments before, during and after the vocational education measure. Planning, execution and evaluation are intended for each core process. A distinguishing feature of the model is the simultaneous build-up and dismantling of behavior: The desired target behavior is encouraged or built up and the actual behavior that is inhibiting the objective is dismantled in the learning process which serves to secure the learning transfer.

A limitation of this study can be seen in the methodology: First, the cluster-analysis does not allow generalizations about the sample. And second, the questionnaire does not include any questions about the concepts of those companies who secure and evaluates the whole transfer chain.

Further research could be conducted to analyze the transfer concepts of the companies which secure the whole transfer chain. Other recommendations for further research include the following: identify which differences in securing learning transfer exist depending on the organizational culture and depending on other levels of culture.

The proposed model has to be applied to the vocational education context. Recommendations for implementing this model are to develop.

Additional research in the field of evaluating the spiral model in the companies will add to the deeper understanding about the impact of this model.

\section{Acknowledgement}

The author would like to thank the anonymous reviewer for careful review of my manuscript and providing me with the comments and suggestion to improve the quality of the manuscript.

\section{References}

[1] American Society for Training and Development. (2003). 2003 State of the industry report. Alexandria, VA: ASTD press.

[2] T.T. Baldwin and J.K. Ford (1988). Transfer of training: A review and directions for future research. PersonnelPsychology, 41(1), 63-105.

[3] K. Bouzguenda (in press). Enablers and Inhibitors of Learning Transfer from Theory to Practice. In Schneider, K. (Ed.) Transfer of Learning in Organizations. Heidelberg,
New York, Dordrecht, London: Springer.

[4] L.M. Broad and W.J. Newstrom (1992). Transfer of training: Action packed strategies to ensure high payoff from training investments. Reading, MA: Addison-Wesley.

[5] J. Heinsenand M. Vollmer (2007). Bildungscontrolling und Transfersicherung. Überblick, Einordnung und Ergebnisse einer empirischen Untersuchung von Erwachsenenbildung und Wirtschaft.[Education Controlling andSecuringthe Transfer. Summary, Categorization and Results of an Empirical Examination of Adult Education and the Economy]. Saarbrücken: VDM Verlag Dr. Müller.

[6] E.F. Holton and T. Baldwin (2003). Making transfer happen: An action perspective on learning transfer systems. In Holton, E.F. and T. Baldwin (Eds.) Improving learning transfer in organizations (pp. 3-15). San Francisco, CA: Jossey-Bass Publisher.

[7] T. R. Hummel (2001). Erfolgreiches Bildungscontrolling. Praxis und Perspektiven [Successful Education Controlling. Practice andPerspectives], 2nd ed. Heidelberg: Sauer-Verlag.

[8] B. Käpplinger (2009). Bildungscontrolling: Vor allem in Großbetrieben ein Thema. BIBB-Umfragen von 1997 und 2008 imVergleich [Education Controlling: An Issue Primarily in Large Companies. Comparison of the Survey by the German Federal Institute for Vocational Education and Training in 1997 and 2008]. BIBB-Report, 13, 1-8.

[9] R. Kegan and L.L. Lahey (2009). Immunity to change. How to overcome it and unlock potential in yourself and your organization.Boston, MA: Harvard Business Press.

[10] C. Kontoghiorghes (in press). A Systemic Perspective of Training Transfer. In Schneider, K. (Ed.) Transfer of Learning in Organizations. Heidelberg, New York, Dordrecht, London: Springer.

[11] D. H.H. Lim and B. Nowell, B. (in press). Integration for Training Transfer: Learning, Knowledge, Organizational Culture, and Technology. In Schneider, K. (Ed.) Transfer of Learning in Organizations. Heidelberg, New York, Dordrecht, London: Springer.

[12] C. Macaulay and H. Loney (2000). Transfer of Learning: A Study.Edinburgh: Scottish Office Central Research Unit.

[13] E. Mooi and M. Sarstedt (2011). A Concise Guide to Market Research. The Process, Data, and Methods using IBM SPSS statistics. Berlin and Heidelberg: Springer.

[14] S. S. Naquin and T. Baldwin (2003). Managing transfer before learning begins. In Holton, E. and T. Baldwin (Eds.) Improving transfer in organizations (pp. 80-96). San Francisco: Jossey-Bass.

[15] R. Noe and N. Schmitt (1986). The influence of trainee attitudes on training effectiveness: Test of a model. PersonnelPsychology, 39(3), 497-523.

[16] P. Pineda-Herrero, C. Quesada-Pallarès, and A. Ciraso-Calí, A. (in press). Evaluation of Training Transfer Factors: The FET Model. In Schneider, K. (Ed.) Transfer of Learning in Organizations. Heidelberg, New York, Dordrecht, London: Springer.

[17] D. D. Pratt (2002). Good Teaching: One Size Fits All? InRoss-Gordon, J. (Ed.) Contemporary Viewpoints on Teaching Adults Effectively. Number 93. (pp. 5-16). San Francisco: Jossey-Bass.

[18] J.-F. Roussel (in press). Learning Transfer in Organizations: An Adaptive Perspective Centered on the Learner and the 
Development of Self-Regulation. In Schneider, K. (Ed.) Transfer of Learning in Organizations. Heidelberg, New York, Dordrecht, London: Springer.

[19] M. A. Saks and R. Haccoun (2004). Managing performance through training and development. Scarborough: Thomson Nelson.

[20] K. Schneider, M. Pältz, and H. Stauche (in press). Transfer of Learning in German Companies. In Schneider, K. (Ed.) Transfer of Learning in Organizations. Heidelberg, New York, Dordrecht, London: Springer.

[21] G. Schraw and D. R. Robinsohn (Eds.) (2011). Assessment of Higher Order Thinking Skills. A Volume in Current Perspectives on Cognition, Learning, and Instruction. Charlotte, NC: Information Age publishing, Inc.

[22] S. Seyda and D. Werner (2012). Umfang, Kosten und Trends der betrieblichen Weiterbildung - Ergebnisse der IW-Weiterbildungserhebung 2008. [Scope, Costsand Trends of Company Training - ResultsoftheEconomicInstitute's 2008 Training Survey] In IW Trends-Vierteljahresschrift zur empirischen Wirtschaftsforschung aus dem Institut der deutschen Wirtschaft Köln39 (1), 1-19.
DOI:10.2373/1864-810X.12-01-03.

[23] R. J. Spiro and M. DeSchryver (2009). Constructivism. When It's the Wrong Idea and When It's the Only Idea. In Tobias, E. and T. M. Duffy (Eds.) Constructivist Instruction. Successorfailure? (pp. 106-123). New York and London: Routledge.

[24] L. J. Splitter (2010). Dispositions in education: Nonentities worth talking about. Educational Theory, 60(2), 203-230.

[25] Statistisches Bundesamt [German Federal Statistical Office] (2013). Berufliche Weiterbildung in Unternehmen. Vierte europäische Erhebung über die berufliche Weiterbildung in Unternehmen [Vocational Training in Companies. Fourth European Survey on Vocational Training in Companies]. (CVTS4) 2010. Wiesbaden: StatistischesBundesamt [German Federal Statistical Office].

[26] S. W. Yoon and D. H. Lim (2007). Strategic blending: A conceptual framework to improve learning and performance. International Journal on E-Learning, 6 (3), 475-489. 